at entry. Risk estimates (RR, HR, OR) from models with the highest degree of multivariate adjustment in each study were transformed to a standardised top-vs-bottom fifth estimate according to the population's baseline distribution of each nutrient's values. We used the $\mathrm{I}^{2}$ statistic to measure heterogeneity between studies and calculated pooled risk estimates for incident diabetes with randomeffects meta-analysis.

Results Ten prospective cohort studies with data on 420840 participants and 11517 incident diabetes events were included. Highest to lowest fifth of intake of sucrose was associated with a $15 \%$ lower risk of diabetes (RR: $0.85,95 \%$ CI 0.75 to 0.97 ). Other carbohydrate subtypes were not significantly associated with diabetes risk

Conclusion All studies reported risk estimates adjusted for total energy intake and thus model an iso-energetic diet. Lower risk of diabetes associated with higher intake of sucrose is most likely to reflect the effect of substitution of sucrose for other nutrients rather than net increased intake of sucrose itself. Nutrient substitution patterns require further investigation.

\section{P2-5 VARIABILITY IN THE CONTROL OF CHRONIC PATIENTS IN PRIMARY CARE ACCORDING TO THE ELECTRONIC CLINICAL RECORD}

doi:10.1136/jech.2011.142976h.42

\begin{abstract}
${ }^{1,2} \mathrm{~F}$ Aizpuru, ${ }^{*}{ }^{3} \mathrm{I}$ Vergara, ${ }^{1} \mathrm{~J} \mathrm{P}$ de Arriba, ${ }^{1,3} \mathrm{~A}$ Latorre, ${ }^{4} \mathrm{~B}$ lbañez, ${ }^{1,3} \mathrm{~K}$ Latorre, ${ }^{1}$ A Apiñaniz, ${ }^{1} \mathrm{R}$ Samper, ${ }^{1} \mathrm{~J}$ L Bilbao. ${ }^{1}$ Osakidetza/Basque Health Service, Basque Country, Spain; ${ }^{2}$ University of the Basque Country, Basque Country, Spain; ${ }^{3}$ BIO Eusko Fundazioa, Basque Country, Spain; ${ }^{4}$ Fundación Miguel Servet, Pamplona, Spain
\end{abstract}

Introduction This study aims to describe the variability in Primary Care to comply with the good practice requirements (GPR) for the management of the following chronic conditions: Hypertension, hypercholesterolaemia, diabetes, alcohol abuse, COPD, depression, dementia, anxiety, asthma and obesity.

Methods The electronic clinical records of all general practitioners (1685; 2147754 professionals) of Osakidetza/Basque Health Service were examined. The rate of compliance of each of the GPR considered by the Health Plan of Basque Autonomous Community of Spain, standardised by age and sex, was calculated, as well as the variability statistics: extremal quotient $\left(\mathrm{EQ}_{5-95}\right)$, coefficient of variation $\left(\mathrm{CV}_{5-95}\right)$ and systematic component of variation $\left(\mathrm{SCV}_{5-95}\right)$.

Results The electronic records show that more than half of the patients are correctly controlled in nine out of the 44 GPR studied. On the contrary, in 16 GPR the compliance rate is lower than $25 \%$ of the diagnosed patients. The smallest variability inter-centres is observed in the management of hypertension, hypercholesterolaemia, obesity and diabetes, all with $\mathrm{SCV}_{5-95}$ $<0.10$. Disparity is moderate in COPD and alcohol abuse $\left(0.10<\mathrm{SCV}_{5-95}<0.20\right)$, high in depression, anxiety, dementia and asthma in adults $\left(0.20<\mathrm{SCV}_{5-95}<0.50\right)$ and very high in asthma in children $\left(\mathrm{SCV}_{5-95} \geq 0.50\right)$.

Conclusion Control of patients diagnosed with chronic processes, especially those with a shorter tradition in Primary Care is insufficient.

\section{P2-6 INFLUENCE OF THE NEIGHBOURHOOD ENVIRONMENT ON WAIST SIZE OVER TIME AMONG IMMIGRANTS TO THE USA: THE MULTI-ETHNIC STUDY OF ATHEROSCLEROSIS}

doi:10.1136/jech.2011.142976h.43

${ }^{1} \mathrm{~S}$ Albrecht, ${ }^{*} \mathrm{~A}$ Diez Roux, ${ }^{2} \mathrm{~L}$ Gallo, ${ }^{3} \mathrm{~N}$ Kandula, ${ }^{4} \mathrm{~T}$ Osypuk, ${ }^{5} \mathrm{H} \mathrm{Ni},{ }^{6} \mathrm{~S}$ Shrager.

${ }^{1}$ University of Michigan, Ann Arbor, Michigan, USA; ${ }^{2}$ San Diego State University, San
Diego, California, USA; ${ }^{3}$ Northwestern University, Chicago, Illinois, USA; ${ }^{4}$ Northeastern University, Boston, Massachusetts, USA; ${ }^{5}$ National Institutes of Health, Bethesda, Maryland, USA; ${ }^{6}$ University of Washington, Seattle, Washington, USA

Introduction Greater time in the USA has been associated with a higher risk of obesity among immigrants. Few studies have examined this pattern longitudinally or considered measures of the neighbourhood environment in evaluating weight-related change among immigrants the longer they live in the USA.

Methods Using prospective data from 883 Hispanic and 688 Chinese foreign-born subjects aged 45-84 in the Multi-ethnic Study of Atherosclerosis, we used linear mixed models to examine whether neighbourhood environments characterised by greater healthy food availability and greater walkability are associated with baseline waist circumference (WC) and with change in WC over a median follow-up of 5 years.

Results Neighbourhoods were characterised using survey items; higher scores represented better environments. Adjusting for age, sex, education, income, years lived in the US at baseline, and neighbourhood poverty, among Hispanics, only greater healthy food availability was associated with lower mean baseline WC (mean difference per SD higher neighbourhood score $=-0.98 \mathrm{~cm}, \mathrm{p}=0.028$ ) There was no association between neighbourhood context and WC change over time. Among Chinese, greater walkability was associated with lower mean baseline WC $(\beta=-1.06 \mathrm{~cm}, \mathrm{p}=0.007)$ and with smaller increases in WC over time (mean difference in annual change per SD higher walkability $=-0.12 \mathrm{~cm}, p=0.003$ ). Associations with walkability also differed for long-term vs more recent immigrants among Chinese. ( $p$ heterogeneity=0.001) (effect modification by baseline length of US residence)

Conclusion Where immigrants reside may have implications for the health patterns that emerge with greater time in the USA.

\section{P2-7 MORTALITY AND CARDIOVASCULAR EVENTS IN PATIENTS UNDER TREATMENT WITH CLOPIDOGREL AND OMEPRAZOLE}

doi:10.1136/jech.2011.142976h.44

V Aliperti, ${ }^{*}$ S Aragone, I Abramovich, S Figar, M Cámera, F G B de Quirós. Hospital Italiano de Buenos Aires, Buenos Aires, Argentina

Introduction In 2009, it was announced that clopidogrel should not be taken with proton pump inhibitors. Omeprazole possibly reduces antiplatelet effect of clopidogrel. We compared mortality and cardiovascular rates between patients that had been treated with clopidogrel alone and those with both clopidogrel and omeprazole.

Methods A retrospective dynamic cohort study using secondary data of a health information system from a Health Maintenance Organization in Buenos Aires was analysed. Patients older than 17 years with purchase record of clopidogrel were followed for allcause mortality and cardiovascular events (CE) from 1 January 2004 to 31 December 2008. Rates and 95\% CIs are expressed per 1000 persons-year. Cox regression was used to obtain adjusted HRs for the risk of all-cause mortality and CE in groups exposed and unexposed concomitant to omeprazole at baseline.

Results Mean follow-up 13 months, 2518 patients received clopidogrel from whom $17.31 \%$ also received omeprazole. Exposed and unexposed to omeprazole were similar in sex (male 60\%), age (mean 68) and comorbidities. The CE rate was 32.4 (95\% CI 27.3 to 38.4) and 26.1 (95\% CI 24.1 to 28.4 ) for each group respectively (RR 1.23 $(\mathrm{p}=0.026)$ and adjusted RR $1.15(\mathrm{p}=0.137))$. The all-cause mortality rate was 2.5 (95\% CI 1.4 to 4.5$)$ and 1.23 (95\% CI 0.8 to 1.7$)$ for each group respectively (RR $2.06(p=0.034)$ and adjusted $R R 1.76$ $(\mathrm{p}=0.109))$. 